\title{
TWO DIMENSIONAL ASPECTS OF REGENERATIVE BBU IN TWO-PASS RECIRCULATING ACCELERATORS *
}

\author{
E. Pozdeyev, JLab, Newport News, VA 23606, USA
}

\begin{abstract}
In this paper, I present the formula, describing a threshold of the regenerative multi-pass Beam Breakup (BBU) for a single dipole higher order mode with arbitrary polarization in a two-pass accelerator with a general-form, $4 \times 4$ recirculation matrix. To illustrate specifics of the BBU in two dimensions, the formula is used to calculate the threshold for the reflecting and rotating optics of the recirculator that can lead to higher threshold currents. Then, I present a mathematical relation between transfer matrices between cavities of the accelerating structure and recirculation matrices for each cavity, which must be satisfied in order to successfully suppress the BBU by reflection or rotation in several cavities.
\end{abstract}

\section{INTRODUCTION}

Regenerative multi-pass BBU arises from interaction of an intense recirculated beam with Higher-Order Modes (HOM) of the accelerating structures. In the JLab FEL Upgrade [1], the regenerative, multi-pass BBU was observed at a beam current of approximately $5 \mathrm{~mA}$. Because BBU imposes an immediate threat to operations of the machine, suppression of regenerative BBU is a task of paramount importance.

As shown by R. E. Rand and T. I. Smith in [2], the threshold of regenerative BBU strongly depends on a form of the $4 \times 4$ recirculation matrix. In their paper, the authors investigated the possibility of suppressing the BBU by means of reflection or $90^{\circ}$-rotation of the beam displacement on the second pass. In the Summer of 2004, an optical reflector was installed in the recirculation pass of the JLab FEL Upgrade. The insertion consists of 5 skew-quadrupoles and reflects the beam displacement respective to the $y=x$ plane [3]. The first experience with the reflector showed an increase of the BBU threshold for offensive modes by a factor of 5 [4].

In this paper, a formula for the BBU threshold for a single dipole HOM with arbitrary polarization in a two-pass machine with a general-form, $4 \mathrm{x} 4$ recirculation matrix is derived. The paper also contains formulas describing evolution of the HOM voltage above and below the threshold. To suppress BBU by optical transformations in a machine consisting of several or many accelerating cavities, the recirculation matrix for each cavity has to be of a form that provides effective BBU suppression. To preserve the form of the recirculation matrix from cavity to cavity, transfer matrices between the cavities have to satisfy a condition that is presented at the end of this paper.

\footnotetext{
${ }^{*}$ Work supported by DOE Contract DE-AC05-84ER40150
}

\section{BBU THRESHOLD IN \\ TWO-DIMENSIONAL SYSTEMS AND HOM VOLTAGE BEHAVIOR ABOVE AND BELOW THE THRESHOLD}

\section{Single-mode approximation}

Simulation results and experimental data presented elsewhere [5] suggest that dipole TM HOMs with a quality factor of $10^{5}-10^{6}$ will limit the maximum recirculated current to the order of tens to hundreds of milliamps. Assuming a quality factor of the order of $10^{5}-10^{6}$ and a typical HOM frequency of the order of $2 \mathrm{GHz}$, one concludes that a typical bandwidth of dangerous HOMs is of the order of $1 \mathrm{kHz}$ to $10 \mathrm{kHz}$.

Imperfections and asymmetrical features of cavities such as couplers violate two-dimensional symmetry of cavity geometry and split frequencies of degenerate dipole modes. A typical separation between two orthogonal polarizations of the same dipole mode in a superconducting RF cavity is of the order of several hundred $\mathrm{kHz}$ to several $\mathrm{MHz}$. Besides, imperfections cause the frequency of HOMs to vary from cavity to cavity. The variation of the HOM frequency from cavity to cavity is also of the order of several hundred $\mathrm{kHz}$ to several MHz.

The probability of overlapping of HOM frequencies is small if the number of cavities is significantly smaller than the ratio of the spread of HOM frequencies to the typical bandwidth of the modes. For example, assuming an HOM bandwidth of $10 \mathrm{kHz}$ and a frequency spread of a few $\mathrm{MHz}$, one can consider modes separately if the number of cavities is of the order of a few tens.

\section{Two-dimensional formula for the BBU threshold}

Steering and misalignment errors lead to a constant offset of the beam centroid relative to the cavity axis on the first pass. On the second pass, the transverse beam displacement consists of two terms: a constant offset due to steering/misalignment errors and an oscillatory term induced by the HOM voltage on the first pass. The constant offset induces the HOM voltage, which is always limited in amplitude. The oscillatory term, on the contrary, constitutes a feedback that can cause regenerative beam breakup and, in theory, an infinite growth of the HOM voltage.

In calculating the threshold of the regenerative BBU, we treat the voltage induced by the oscillatory term on the second pass as a perturbation and assume that the HOM voltage is a harmonic function of time. A variation of the energy stored in a dipole TM HOM produced by a point-like bunch, $\Delta U$, is given, to the first order in the bunch charge, 
by [6]

$$
\Delta U=-q \frac{V_{a}}{a} \cos (\phi)(x \cos (\alpha)+y \sin (\alpha)),
$$

where $V_{a}$ is the accelerating HOM voltage at the radius of the beam pipe $a$ induced by previous bunches, $\phi$ is the phase of the point-like bunch with respect to the peak of the HOM electric field, $q$ is the bunch charge, $(x, y)$ is the bunch displacement vector, and $\alpha$ is the mode polarization angle in the $x y$ coordinate system.

The energy deposited by the bunch in the HOM on the first and second passes can be written as

$$
\begin{array}{r}
\Delta U_{1}=-q \frac{V_{a}}{a} \cos (\phi)\left(x_{1} \cos (\alpha)+y_{1} \sin (\alpha)\right), \\
\Delta U_{2}=-q \frac{V_{a}}{a} \cos \left(\phi+\omega T_{r}\right)\left(x_{2} \cos (\alpha)+y_{2} \sin (\alpha)\right),
\end{array}
$$

where $T_{r}$ is the recirculation time. It is assumed in (2-3) that the voltage amplitude $V_{a}$ does not change significantly during recirculation. Exactly at threshold, $V_{a}$ is constant. Coordinates of the recirculated bunch, $x_{2}$ and $y_{2}$, can be expressed via the bunch coordinates before deflection and the HOM accelerating voltage on the first pass as

$$
\begin{gathered}
x_{2}=m_{11} x+m_{12} x_{1}^{\prime}+m_{13} y_{1}+m_{14} y_{1}^{\prime}- \\
-\frac{q V_{a}}{\omega a p} \sin (\phi)\left(m_{12} \cos (\alpha)+m_{14} \sin (\alpha)\right), \\
y_{2}=m_{31} x+m_{32} x_{1}^{\prime}+m_{33} y_{1}+m_{34} y_{1}^{\prime}- \\
-\frac{q V_{a}}{\omega a p} \sin (\phi)\left(m_{32} \cos (\alpha)+m_{34} \sin (\alpha)\right),
\end{gathered}
$$

where $p$ is the beam momentum.

The energy balance equation for the HOM stored energy is

$$
\dot{U}=\dot{U}_{\text {beam }}-P_{c}=\left\langle\Delta U_{1}+\Delta U_{2}\right\rangle \cdot f_{b}-P_{c} .
$$

The averaging is done respectively to the phase of the HOM, $\phi$, taken at moments when bunches pass through the cavity on the first pass. Ohmic losses in the cavity can be expressed as [6]

$$
P_{c}=\frac{V_{a}^{2}}{\left(\frac{\omega}{c}\right)^{2} a^{2}\left(\frac{R_{d}}{Q}\right) Q} .
$$

Terms proportional to $\cos (\phi), \sin (\phi), \cos \left(\phi+\omega T_{r}\right)$, and $\sin \left(\phi+\omega T_{r}\right)$ yield zero after averaging if $x_{1}, x_{1}^{\prime}$ and $y_{2}, y_{2}^{\prime}$ in (4-5) are steering/misalignment errors. If the HOM frequency is not equal to a harmonic of the bunch repetition rate, terms proportional to $\cos (\phi) \cdot \sin (\phi)$ also yield zero and the average value of the $\sin ^{2}(\phi)$ is equal to $1 / 2$. Thus, Equation (6) can be rewritten as

$$
\begin{array}{r}
\dot{U}=I_{b} \frac{q V_{a}^{2}}{\omega p a^{2}} m_{12}^{*}\left\langle\sin (\phi) \cos \left(\phi+\omega T_{r}\right)\right\rangle \\
-\frac{V_{a}^{2}}{(\omega / c)^{2} a^{2}\left(R_{d} / Q\right) Q} \\
=-I_{b} \frac{q V_{a}^{2}}{\omega p a^{2}} m_{12}^{*} \frac{\sin \left(\omega T_{r}\right)}{2}-\frac{V_{a}^{2}}{(\omega / c)^{2} a^{2}\left(R_{d} / Q\right) Q},
\end{array}
$$

where

$$
\begin{array}{r}
m_{12}^{*}=m_{12} \cos ^{2}(\alpha)+\left(m_{14}+m_{32}\right) \sin (\alpha) \cos (\alpha) \\
+m_{34} \sin ^{2}(\alpha) .
\end{array}
$$

At threshold, $\dot{U}$ is equal to zero. Thus, the threshold current is given by

$$
I_{\mathrm{th}} \frac{m_{12}^{*}}{2 p} \frac{q}{\omega} \sin \left(\omega T_{r}\right)+\frac{1}{(\omega / c)^{2}\left(R_{d} / Q\right) Q}=0,
$$

which yields the threshold current as

$$
I_{\mathrm{th}}=-\frac{2 p c}{q \frac{\omega}{c}\left(\frac{R_{d}}{Q}\right) Q m_{12}^{*} \sin \left(\omega T_{r}\right)} .
$$

For positive values of the product $m_{12}^{*} \sin \left(\omega T_{r}\right)$, Equation (11) yields a negative threshold, implying a stable beam. However, the beam can be unstable at extremely high values of the beam current even if $m_{12}^{*} \sin \left(\omega T_{r}\right)>0$. This discrepancy is caused by the assumption that the voltage induced by the beam on the second pass is a small perturbation to the HOM voltage, which fails at high beam intensities. In one-dimensional cases, similar dependence of the threshold on $m_{12} \sin \left(\omega T_{r}\right)$ was predicted analytically and observed in simulations in early works by $\mathrm{G}$. A. Krafft, J. J. Bisognano, and S. Laubach [7] and later by G. H. Hoffstaetter and I. V. Bazarov [8].

\section{Evolution of the HOM voltage below and above the threshold}

Equation (8) can be rewritten as

$$
\frac{d U}{U}=-d t \frac{\omega}{Q} \frac{I_{\mathrm{th}}-I}{I_{\mathrm{th}}} .
$$

The solution of the last equation is

$$
U=U_{0} \exp \left(-t \frac{\omega}{Q} \frac{I_{\mathrm{th}}-I}{I_{\mathrm{th}}}\right) .
$$

The HOM voltage depends on time as

$$
V=V_{0} \exp \left(-t \frac{\omega}{2 Q} \frac{I_{\mathrm{th}}-I}{I_{\mathrm{th}}}\right) .
$$

Equations (13) and (14) describe evolution of the stored energy and HOM voltage below and above the threshold. Using equations (13) and (14), one can introduce the effective quality factor $Q_{\text {eff }}$ for the beam-HOM system, given by the equation

$$
Q_{\mathrm{eff}}=Q \frac{I_{\mathrm{th}}}{I_{\mathrm{th}}-I} .
$$

Equation (15) can be also rewritten in terms of the decay time as

$$
\tau_{\mathrm{eff}}=\tau \frac{I_{\mathrm{th}}}{I_{\mathrm{th}}-I} .
$$


Above the threshold, the decay time given by (16) changes its sign and becomes the growth time of the instability.

Equations (13-16) are also valid for negative values of the threshold current given by (11) for $m_{12}^{*} \sin \left(\omega T_{r}\right)>0$ as long as the beam current is not too high and the assumptions made during the derivation of (11) are valid. For negative values of the BBU threshold given by (11), Equation (15) can be rewritten as

$$
Q_{\mathrm{eff}}=Q \frac{-\left|I_{\mathrm{th}}\right|}{-\left|I_{\mathrm{th}}\right|-I}=Q \frac{\left|I_{\mathrm{th}}\right|}{\left|I_{\mathrm{th}}\right|+I} .
$$

The last two equations show that for $m_{12}^{*} \sin \left(\omega T_{r}\right)>0$ the effective quality factor decreases with the beam current.

\section{SUPPRESSION OF REGENERATIVE BBU BY OPTICAL TRANSFORMATIONS IN SEVERAL CAVITIES OF A TWO-PASS ACCELERATOR}

Recirculation matrices for two cavities in a two passmachine $M_{1}$ and $M_{2}$ are related to each other via transfer matrices between the cavities on the first and the second pass, $T_{1}$ and $T_{2}$ respectively, as

$$
M_{2}=T_{2} M_{1} T_{1}^{-1} .
$$

If $M_{1}$ and $M_{2}$ are of a form that provides effective suppression of the BBU, Equation (18) yields a condition on the form of the transfer matrices $T_{1}$ and $T_{2}$, which has to be satisfied for the particular form of the recirculation matrices $M_{1}$ and $M_{2}$. Using the fact that the transfer matrices are symplectic, one can transform the Equation (18) into

$$
M_{2}=-T_{2} M_{1} S T_{1}^{T} S .
$$

The last equation also allows one to express the condition (18) as a set of equations for $2 \times 2$ block sub-matrices of $M_{1,2}, T_{1,2}$, and $S$.

Using (18), it is trivial to show that, if $M_{1}$ and $M_{2}$ are fully coupled $4 \times 4$ matrices exchanging the $x$ and $y$ betatron planes and all HOMs are bound to the horizontal and vertical planes, all uncoupled transfer matrices $T_{1,2}$ satisfy Equation (18) :

$$
\begin{gathered}
M_{2}=T_{2} M_{1} T_{1}^{-1} \\
=\left[\begin{array}{cc}
X_{2} & 0 \\
0 & Y_{2}
\end{array}\right]\left[\begin{array}{cc}
0 & A \\
B & 0
\end{array}\right]\left[\begin{array}{cc}
X_{1}^{-1} & 0 \\
0 & Y_{1}^{-1}
\end{array}\right] \\
=\left[\begin{array}{cc}
0 & X_{2} A Y_{1}^{-} 1 \\
Y_{2} B X_{1}^{-1} & 0
\end{array}\right] .
\end{gathered}
$$

Thus, an optical insertion interchanging the vertical and horizontal planes installed in the recirculation path can simultaneously suppress HOMs in different cavities if the HOMs are bound to the horizontal and vertical planes.

\section{CONCLUSIONS}

Equation (11), describing the BBU threshold for a single dipole HOM with arbitrary polarization and a generalform, $4 \times 4$ recirculation matrix in a two-pass accelerator, has been derived. The equation can be used to estimate the BBU threshold in the case of several high- $Q$ HOMs if the separation between frequencies of the HOMs exceeds the bandwidth of the modes.

Reflection or rotation produced by the recirculation optics can significantly suppress or completely eliminate the regenerative BBU. This result was first predicted by R. E. Rand and T. I. Smith in [2] and confirmed in this work analytically.

To provide BBU suppression by reflection or rotation simultaneously in several cavities of the accelerating structure, the transfer matrices between the cavities have to satisfy equation (18).

If HOMs are bound to the horizontal and vertical planes, BBU can be simultaneously suppressed in several cavities by an optical transformation with a matrix of the form

$$
\left[\begin{array}{cc}
0 & A \\
B & 0
\end{array}\right]
$$

where $A$ and $B$ are $2 \times 2$ block matrices. An optical insertion providing the $x-y$ motion exchange can consist of solenoids and/or skew quadrupoles and can be located in the recirculation path of a two-pass machine.

\section{ACKNOWLEDGMENTS}

I would like to thank Geoffrey Krafft, Lia Merminga, Todd Smith, Chris Tennant, and Byung Yunn for their interest in the work and useful suggestions. This work was supported by the U.S. Department of Energy under Contract No. DE-AC05-84ER40150.

\section{REFERENCES}

[1] S.V. Benson, et al., "High Power Lasing in the IR Upgrade FEL at Jefferson Lab ", Procceedings of FEL 2004 Conference, Trieste (2004)

[2] R.E. Rand and T.I. Smith, Particle Accelerators, V.11, 1 (1980)

[3] D. Douglas, JLAB Tech. Rep. TN-04-016 (2004)

[4] C. Tennant et al., "Experimental Investigation of Beam Breakup in the Jefferson Laboratory $10 \mathrm{~kW}$ FEL Upgrade", these proceedings.

[5] C. Tennant, Pozdeyev, S. Simrock, A. Sun and H. Wang, JLAB Tech. Rep. TN-04-008, (2004)

[6] H. Padamsee, J. Knobloch, T. Hays, "RF Superconductivity for Accelerators", John Wiley \& Sons, Inc. (1998)

[7] G.A. Krafft, J.J. Bisognano, S. Laubach, unpublished (1987)

[8] G.H. Hoffstaetter, I.V. Bazarov, Phys. Rev. ST Accel. Beams, V7, 054401, 2004 